\title{
Poética de la decadencia y filosofía del nihilismo*
}

The poetics of decadence and the philosophy of nihilism

\author{
Gonzalo Portales
}

\begin{abstract}
Universidad Austral de Chile, Facultad de Filosofía y Humanidades, Instituto de Filosofía y Estudios Educacionales, Valdivia, Chile. Correo electrónico: gportale@uach.cl
\end{abstract}

El artículo se refiere principalmente a la recepción de la obra de Baudelaire en el pensamiento tardío de Nietzsche en el que se propone una filosofía del nihilismo europeo. Siguiendo el hilo conductor del concepto de decadencia, el trabajo vincula los aportes estéticos de la poética francesa y la filosofía alemana del fin de siècle.

Palabras clave: Nietzsche, Baudelaire, poesía, filosofía, nihilismo.

This paper refers mainly to the reception of Baudelaire's work in Nietzche's late thought in which a philosophy of the European nihilism is proposed. Following the train of thought of the concept of decadence, this study links the aesthetic contribution of French poetry to the German philosophy of the fin de siècle.

Key words: Nietzsche, Baudelaire, poetry, philosophy, nihilism.

En el contexto de una meditación sobre la historia que culminaría algo más tarde con la composición de la segunda de sus Consideraciones Intempestivas, el joven Nietzsche registra en sus cuadernos la frase "se necesita mucho tiempo hasta que un mundo desaparece, pero nada más que eso" (KSA 7, 693)1 como si se tratase de una cita exacta de Edward Gibbon en su célebre The history of the decline and fall of the Roman Empire (1776-1788). Aun cuando se ha constatado que tal expresión no se encuentra así textualmente en la monumental obra sobre la decadencia del imperio romano, ${ }^{2} \mathrm{su}$ atribución permite suponer una aprobación al menos genérica de la interpretación de este deterioro a partir del siglo III y, sobre todo, de la tesis central que afirma con carácter de necesidad el ocaso de un poder o de una cultura mediante el inexorable paso del tiempo.

* Este trabajo es resultado parcial del proyecto Fondecyt $\mathrm{N}^{\circ} 1090379$.

Friedrich Nietzsche Kritische Studienausgabe (=KSA). Editada por Giorgio Colli y Mazzino Montinari. Berlín- New York 1988.

2 El trabajo filológico de la edición crítica confirma que no se trata en esta expresión de una cita de Gibbon (cf. KSA 14, 551), sino sólo de una síntesis de significado. 
Esta perspectiva, que privilegia la imagen mítico-literaria de Cronos devorando a sus hijos, se vuelve paradigmática cuando se realiza la comparación que muestra cómo mientras en la antigüedad tardía la experiencia inmediata de la devastación de Roma debía ser aún comprendida por una teología política que operaba dentro de los límites asignados por la historia soteriológica y su misterio partía del hecho de que para ella es "la divina providencia la que constituye totalmente los reinos humanos ("prorsus divina providentia regna constituuntur humana"), y que, por lo tanto, no se debiese creer que lo que sucede en dichos reinos pudiese encontrarse fuera de la voluntad que mueve las leyes providenciales (nullo modo est credendus regna hominum ... a suae providentiae legibus alienas esse voluisse); ${ }^{3}$ muy por el contrario, la moderna filosofía de la historia intenta más bien explicar dichos acontecimientos a partir de las lois naturelles de los órdenes políticos y sus modificaciones, como se desarrolla, por ejemplo, en la obra Considérations sur les causes de la grandeur des Romains et de leur décadence (1734) de Montesquieu, en directa controversia con las interpretaciones formuladas por autores como Maquiavelo y Bossuet, quienes -desde puntos de vistas a su vez divergentes- compartían sin embargo la idea de una temporalidad que no podía ya ser considerada como una mera referencialidad de determinada facticidad política, sino que pasaba a constituir el acontecimiento mismo del devenir histórico.

En su obra tardía, Nietzsche proclamará su empatía intelectual con estos rasgos de la "modernidad" cuando confiesa su predilección por Maquiavelo y Tucídides como posibilidad de cura contra el platonismo y sus pretensiones de atemporalidad eidética a la que oponen con fuerza un instinto de realidad (cf. KSA 13, 615). Pero en sus inicios, la cuestión de la caducidad no surge para Nietzsche sólo como un problema referido a la comprensión del cambio histórico o de las (aunque en ocasiones largo tiempo estables, en definitiva siempre transitorias) organizaciones culturales, sino principalmente en relación al arte, es decir, como una interrogación estética vinculada, por cierto, a la reflexión filológica. Parece interesante advertir, en primer término, que en la obra temprana aún no se ha integrado el galicismo décadence, propio de la reflexión sobre el nihilismo en los escritos del último Nietzsche, sino que la decadencia en el arte es señalada todavía en esta época mediante el concepto alemán Verfall y su amplio ámbito referencial que puede ir desde el estado de ruina de una construcción hasta la pérdida del vigor vital del cuerpo o aludir a un excesivo relajo de las costumbres recriminado por la autoridad moral. De este modo, el joven Nietzsche escribe: "La decadencia de la tragedia, tal como creyó verla Eurípides, era una fantasmagoría socrática. Puesto que nadie podía trasladar satisfactoriamente a palabras y conceptos la sabiduría de la antigua técnica artística, Sócrates negó esta sabiduría, y con él la negó el seducido Eurípides" (KSA 1 542). Coincidiendo, al menos, con lo que la filología romántica de los hermanos Schlegel había anteriormente determinado como origen, desarrollo y fin de la escritura trágica de la antigüedad (cf. Behler 1993), pues no se puede documentar una lectura directa de los textos de la Escuela de Jena en que se describe este transcurso, Nietzsche sostiene que "el momento culminante y supremo del drama musical es Esquilo en su primer gran período", mientras que ya con Sófocles "comienza la paulatina decadencia (der ganz allmähliche Verfall) hasta que finalmente Eurípides... puso fin al proceso con celeridad de tormenta" (KSA 1, 549).

Agustín; De civitate Dei, lib. V, cap. 1. 
Sin embargo, un breve fragmento "programático" de esta obra temprana (cf. KSA 7, 12-13), permite, a mi juicio, advertir que este transcurso -que se desplaza desde la vitalidad originaria del arte trágico y su fundamento en la fiesta dionisíaca hasta su ocaso en la fijación formal del género literario y su respectiva afiliación canónica- se encuentra asociado a un proceso más amplio y complejo de agotamiento de la cultura griega en la medida en que ella - así lo interpretará gran parte de la graecomanía decimonónica-se distancia paulatinamente de sus raíces religioso-poéticas. Se constata, pues, un movimiento que bien podría ser entendido al modo de un tránsito contrario al que se enuncia en la anhelada plenitud de la obra de arte total o Gesamtkunstwerk y que el texto señala mediante la expresión desintegración de las artes (Auseinanderfallen der Künste), identificando tal acontecimiento temporalmente con la época de Sófocles en la que "el arte absoluto", junto con manifestar su culminación, habría señalado también de manera simultánea la decadencia de las prácticas estéticas, revelando así la inevitable ambigüedad de su condición, la que se deja presentar a través del símil de un árbol que ya no es capaz de sostener sus propios frutos. ${ }^{4} \mathrm{El}$ fragmento finaliza su enumeración programática con lo que parece ser una explicación ejemplar destinada a ilustrar sucintamente el punto preciso en que se habría desencadenado la decadencia de las artes en la antigüedad, esto es, el instante exacto en que "la poesía se vuelve política, discurso." En ese momento, agrega Nietzsche en un tono que no deja muchas incertidumbres acerca de su desconsuelo, es cuando "comienza el reino de la prosa." Un reino, habría que añadir, en el que la obra trágica ya no puede tener cabida, pues sus personajes - como se manifiesta de modo paradigmático en los dramas de Eurípides- se ven forzados a abandonar sin más el habla poética de la creación literaria para admitir en su reemplazo el discurso apodíctico de la prosa política. También la prosa filosófica habría contribuido con su parte propia a esta ruina del arte, especialmente a partir de la dialéctica socrática y su fastidiosa obstinación por "vencer" en la disputa dialógica. En tiempos anteriores, se precisa, esa misma prosa convivía aún armoniosamente con la poíesis, generando una consonancia que puede percibirse todavía, piensa Nietzsche, en las magníficos escritos de Heráclito, Demócrito y Empédocles (Cf. KSA 7, 13). ${ }^{5}$

Aun cuando es innegable tanto la gran pasión científica de estos primeros estudios de la tragedia griega, como también el apremio vital involucrado en el desarrollo de esta filología de la decadencia, parece evidente, sin embargo, que ella se encuentra de algún modo protegida de las secuelas más nocivas de ese proceso de declinación cultural y lo observa desde un relativo amparo otorgado por aquella distancia epocal en la que los residuos no sólo señalan un tiempo pretérito, sino que indican asimismo un pasado que ya tuvo su fin, es decir, un suceso completamente acaecido con el que, no obstante cierta ilusión decimonónica, no se puede tener ya de manera genuina ninguna filiación. La ruina estética se constituye así inevitablemente en objeto de una philologia clásica que se ve siempre forzada a acometer la disección del corpus yaciente de una lengua muerta que relata el ocaso de la fiesta orgiástica mediante su incorporación al canon tradicional de una literatura patrocinada por el Estado (Polis).

"Zeitpunkt vor Sophokles: die absolute Kunst ist Anzeichen, daß der Baum die Früchte nicht mehr halten kann"

El aparato de la edición crítica privilegia en la interpretación del pasaje final del fragmento ("Früher selbst in der Prosa die Poesie. Heraclit, die Pythia. Democrit. Empedocles”) la referencia a Heráclito, señalando una alusión indirecta al fragmento 92 de la edición Diels-Kranz en la que se compara la poesía de Safo con la retórica profética de la Sibila (cf. KSA 14, 529). 
El inmenso riesgo al que se expone, en cambio, la reflexión tardía sobre la decadencia se explica, a mi juicio, principalmente, por el hecho de que ella se halla justo en medio de la misma facticidad que interroga e interpela y que Nietzsche reconoce como el tiempo del gran peligro o de la historia del nihilismo europeo. En uno de sus últimos escritos, en aquél redactado durante el año 1888 en que la disputa con Wagner se presenta como "un problema para músicos", se puede leer el siguiente pasaje testimonial: "Lo que más a fondo me ha ocupado" - dice allí- "ha sido de hecho el problema de la décadence - he tenido motivos para ello (...) Si se ha adquirido buen ojo para percibir los signos de la caída (Niedergang), se comprenderá también la moral - se comprenderá lo que se oculta bajo sus más sagradas denominaciones y fórmulas valóricas: la vida empobrecida, la voluntad de tener un final, el gran cansancio (...) Para una tarea semejante me fue necesaria la autodisciplina: -Tomar partido contra todo lo que había de enfermo en mí, incluido Wagner, incluido Schopenhauer, incluido todo el 'humanitarismo' moderno" (KSA 6, 11-12).

Una primer atisbo del contenido de esta confidencia permite advertir que en la escritura del Nietzsche tardío se distingue el uso de la expresión francesa décadence como terminus technicus, aunque en ocasiones se equipare todavía sin más con la noción alemana de Verfall $^{6}$, para designar un modo específico de la decadencia, el que ya no se inscribe solamente en la posible descripción del ocaso de una cultura originaria por medio de la atenuación de la potencia emancipadora del arte, sino que su significado apunta ahora directamente a un agotamiento de las condiciones básicas de pervivencia del hombre (Mensch) europeo y anuncia en consecuencia el advenimiento del nihilismo. Tal contexto había sido ya expuesto de forma manifiesta por la Genealogía de la moral (1887) al desenmascarar sin contemplaciones el secreto de la pretendida sacralidad de una antropología de la compasión cuyo artificio no había hecho sino despojar al hombre de toda aspiración de grandeza, precipitándolo a un estado de mera indiferencia en el que se busca con afán su empequeñecimiento y nivelación (Verkleinerung und Ausgleichung) político-económica (cf. Müller-Lauter 1999, 200 y ss.). A la pregunta por el sentido actual del nihilismo, se responde allí con el enunciado que expresa la fatalidad de Europa: "Estamos cansados de el hombre..." (KSA 5, 278). ${ }^{7}$ El "gran cansancio" en que se funda toda clase de humanitarismo (en este caso, decimonónico) se manifiesta, pues, como una nueva versión de la fatalidad romana del taedium vitae, sólo que esta vez se constituye en un taedium mundi $i^{8}$ que se consume en una "melancolía de las ruinas" (KSA 3, 69) y sus efectos se asemejan a la aparición de un "budismo-europeo" cuya culminación es precisamente el nihilismo contemporáneo como obra política, literaria y artística de ese continente (cf. KSA 6, 252 y Gawoll 1989, 199 y ss.).

Sin embargo, esta suma de "reparos" no debe entenderse al modo de un lamento patético y moralizante sobre el nefasto momento de la cultura occidental a causa de una supuesta traición y abandono de principios tradicionales que se encontrarían en sus más íntimas raíces y que, por lo mismo, reclamarían fidelidad. Por el contrario,

\footnotetext{
$6 \quad$ Así, por ejemplo, cuando en el Crepúsculo de los ídolos (KSA 6, 138) se refiere a los efectos de la décadence en las ciencias particulares y reprocha a la sociología el tener experiencia sólo de formas-decadentes (VerfallsGebilde) o en El Anticristo (KSA 6, 185), en donde equipara Verfalls-Gebilde a Décadence-Instinkte para remitirse al "deplorable Dios del monótono-teísmo".

"Hier eben liegt das Verhängniss Europa's (...) Wir sind des Menschen müde..."

8 Cf. KSA 3, 55: "Welt-Müdigkeit".
} 
Nietzsche nunca reivindica para su crítica un topos privilegiado por medio del cual pudiera situarse con derecho fuera de la decadencia que ella misma describe, como si se hallase liberada de sus efectos y se alzara incólume por sobre la abrumadora y espectral incertidumbre que recorre Europa; y menos pretende aún calificar el estado de postración de dicha cultura como consecuencia de una pérdida de preciados orígenes que debiesen ser restaurados. Así, al mismo tiempo en que los textos pertenecientes a la época de la filosofía del nihilismo europeo exponen un despiadado diagnóstico del estado de cosas pertenecientes al fin de siècle, se permiten, no obstante, el juego irónico de la ambigüedad, la que si bien no puede privar de toda su amenaza a la situación descrita, sí permite al menos despojar de cualquier prerrogativa a quien la describe: "Descontado, pues, que soy un décadent, soy también su antítesis", declara Nietzsche en las primeras páginas de Ecce homo, y prosigue: "Mi prueba de ello es, entre otras, que siempre he elegido instintivamente los medios correctos contra las malas condiciones; en cambio, el décadent en sí elige siempre los medios que lo perjudican. Como summa summarum yo estaba sano; como ángulo, como especialidad, yo era décadent" (KSA 6, 266).

Este pasaje parece ser decisivo, a mi juicio, cuando se intenta determinar con más precisión tanto el ámbito semántico como así también el sentido político-estético involucrados en el tardío concepto nietzscheano de décadence. El primer indicio de importancia para esta tarea lo otorga ya, sin duda, como se ha señalado anteriormente, el uso del propio galicismo, en el que se refleja la actitud de una resuelta opción por la cultura francesa que Nietzsche ha proclamado con cierto desenfado anti-germánico en un célebre pasaje de esta "obra autobiográfica". ${ }^{9}$ En segundo lugar, creo que se puede afirmar que el origen de la contradicción de quien se asigna a sí mismo de manera simultánea el atributo de la decadencia y de su antítesis sugiere con claridad una alusión a la figura de Baudelaire, tal como Nietzsche comienza a integrarla a su reflexión sobre el nihilismo perteneciente a los últimos años productivos y que ha sido expuesta con prolijidad histórico-filológica por Karl Pestalozzi en su Nietzsches Baudelaire-Rezeption (1978). En lo que se refiere específicamente al paradojal tópico de la atribución antitética de la condición de décadent, se muestra además una manifiesta influencia del capítulo dedicado a Baudelaire en el Essai de psychologie cotemporaine de Paul Bourget, que Nietzsche lee con gran entusiasmo en ese mismo período. ${ }^{10}$ Allí se describe la experiencia vital y poética de Baudelaire como un apremio que lo fuerza a transitar desde el estado acorde a un homme de décadence hasta el de la lucidez propia de un théoricien de décadence. ${ }^{11}$

9 Cf. Ecce homo en: KSA 6, 285. Luego de advertir que de los pocos casos de cultura elevada que ha encontrado en Alemania, todos tenían procedencia francesa (französische Herkunft), Nietzsche declara su amor por Pascal y su admiración por Montaigne, Corneille y Racine, entre otros.

10 Así lo expresan varias cartas de su Correspondencia. Cf., por ejemplo, la dirigida a Resa von Schirnhofer en París el día 11 de marzo de 1885, en la que nombra expresamente el Essai de Bourget, a quien califica de discípulo heredero de la genialidad de Stendhal (KGB 7,18). Comentarios sobre esta lectura se encuentran también en varias cartas enviadas a Heinrich Köselitz, Hippolyte Taine y Malwida von Meysenburg, cf. KGB 8, 96, 106 y 447. El Essai fue publicado el año 1881 y Nietzsche lee una edición del año 1883. Se trata en realidad de una serie de retratos de figuras artísticas e intelectuales de la Francia de la época y de comentarios a algunas de sus obras desde una perspectiva que se califica a sí misma de psicología, en un sentido muy cercano al uso de este término en el propio Nietzsche.

11 Bourget (1883, 23-24): "Il était un homme de décadence, et il s'est fait un théoricien de décadence. C'est peut-être là le trait le plus inquiétant de cette inquiétante figure. C'est peut-être celui qui exerce la plus troublante séduction sur une âme contemporaine." 
A partir del análisis de algunos poemas consagrados a las pasiones del eros del libro Les Fleurs $d u$ Mal, expuesto y comentado en el segundo parágrafo de la primera parte del ensayo, Bourget cree poder determinar con exactitud lo que denomina un style de décadence ${ }^{12}$ en la poética de Baudelaire (p. 25), el cual se dejaría describir fielmente como un extraño sincretismo de mística y libertinaje. ${ }^{13} \mathrm{~A}$ la inusual mezcla entre Madonna y Venus que caracteriza a esas femmes de plaisir del poema CIII de la famosa obra lírica, titulado El crepúsculo matutino, y que tiene un cierto paralelo en el Tannhäuser de Wagner, ${ }^{14}$ habría que añadir el texto de Una mártir (CX), la pieza más bella de la colección (la plus belle pièce du recueil), según piensa Bourget, la que permite adivinar, sin embargo, a un creador de visiones depravadas que se extienden hasta el sadismo (visions dépravées jusqu'au sadisme troublent) y que bien podrían repetir el lema que el autor de Philosophie dans le boudoir quería poner sobre el dintel del portal de su pequeña casa de ensueños: “¡Aquí se tortura!” (cf. Bourget 1883, p. 7). Esta controvertida poética del horror, que autoriza a Baudelaire a introducirse hasta la enigmática intimidad erótica del crimen y observar con distancia descriptiva el sufrimiento de la mutilación del cuerpo martirizado por la inmensidad de un deseo que no vacila en llegar hasta la necrofilia para consumar su satisfacción, no pareciera haber producido en Nietzsche el efecto de repugnancia prácticamente unánime experimentado por una hipotética mayoría social, pues de ello lo habría eximido con toda probabilidad el hecho de que en su investigación sobre los prejuicios morales se había encontrado también ya con aquello que describe como "el placer de la crueldad" y su naturaleza catártica en la que se libera asimismo la inmensidad de un deseo público que se complace en el dolor del estigmatizado, extendiendo su pertenencia histórico-antropológica a lo que allí se denomina "el más antiguo regocijo festivo de la humanidad" (cf. KSA 3, 30). Pero lo que más le interesa del trabajo de Bourget, no parece ser, según mi opinión, el enunciado de esta sugerente mixtura entre sacralidad y transgresión, implicada de manera esencial en la ars poetica de Baudelaire y en aquella praxis que le permite observar la decadencia desde un ángulo especialista como experiencia propia, sino que su lectura del Essai se concentra más bien en los parágrafos posteriores a estos "análisis del eros", es decir, en aquellos que Bourget dedica al examen del pesimismo y de la teoría de la decadencia.

Ya los meros títulos de estos acápites nos muestran la estrecha concordancia temática que Nietzsche descubre entre los problemas de su propia obra y los expuestos en este sui generis tratado de psicología y que bien podría explicar en parte la exaltación manifestada en la recepción de algunas de sus tesis, lectura que se lleva a cabo además justamente durante los años dedicados a la producción de una filosofía del nihilismo europeo. En el primero de estos capítulos se desarrolla una

12 Nietzsche toma directamente la expresión estilo de la decadencia, citando incluso la página correspondiente del libro de Bourget, para aplicarla al arte de de Wagner en un fragmento redactado en el invierno boreal de 1883-1884, allí traduce al alemán el término dédadence (Verfall), cf. KSA 10, 645, en cambio en el texto de El caso Wagner, conserva la palabra francesa, cf. KSA 6, 27.

13 Compárense los siguientes textos: "Baudelaire est tout à la fois, dans ses vers d'amour: mystique, libertine et analyseur" (Essai p.5) y la carta a Kóselitz del 26 de febrero de 1888 (KSB 8, 263): "Baudelaire ist libertin, mystisch, 'satanisch', aber vor allem Wagnerisch".

14 Cf. KSA 12, 473, en donde Nietzsche comenta la elección entre Venus y una virgen de Thüringen que debe tomar el personaje de Tannhäuser. 
taxonomía de los diversos modos en que los pueblos europeos habrían reaccionado frente a la desaparición de los fundamentos que el refinamiento civilizatorio otorgaba ilusoriamente a sus sociedades, produciéndose en el continente lo que el texto llama une nausée universelle y que habría tomado la forma de nihilismo entre los eslavos, de pesimismo en los germanos y de neurosis para los latinos (cf. 15). Mediante una enigmática adjudicación del poeta a los síntomas pertenecientes al segundo de estos grupos, Bourget cree poder explicar lo que prefiere denominar "el pesimismo parisino" de Baudelaire a partir de un pronunciado menoscabo de la fe religiosa adquirida desde los años infantiles en las prácticas del catolicismo francés. Para ello establece una comparación entre aquella carencia padecida por los que practican una fe burguesa o superficial, en la que la "muerte de Dios" - para decirlo con palabras de Nietzsche- encuentra un efectivo atenuante en el reemplazo de esa creencia por una fe en la libertad, el orden social, la revolución o la ciencia, con aquella otra pérdida experimentada por un alma mística -como la de Baudelaire- en la que lo abandonado ya no admite ser reemplazado de ninguna manera y se caracteriza más bien por exhibir con desconcertante insistencia el vacío que provoca su ausencia. Es decir, se estaría frente a una "muerte de Dios" que no puede encontrar ningún sustituto y menos aún algún tipo de consuelo, pues, como ejemplifica Bourget aludiendo a conocidas experiencias de Baudelaire: "cuando se ha conocido la embriaguez del opio, la del vino produce aversión y parece mezquina" (cf. 19-20). En este sentido, se interpreta la desesperanza que marca la biografía y la obra de quien se afirma provenir de una estirpe condenada a la infelicidad (condamnée au malheur) como una consecuencia directa de su adhesión a la tradición del pesimismo y que en este caso se fundamentaría además en el siguiente principio que el texto califica de teorema ético: "La fuerza por la que perseveramos en la existencia es limitada y el poder de las causas exteriores la sobrepasa infinitamente..."15

Si se atiende a la ejemplaridad ofrecida por el Essai para ilustrar algunas manifestaciones del pesimismo en su historia decimonónica, entonces se percibe con claridad que las nefastas consecuencias de un axioma de este tipo debiesen ser extendidas, de acuerdo a lo que sostiene allí Bourget, no sólo hasta el fundamento del terror político que emerge de la rabia asesina de los conspiradores de San Petersburgo y a la furia incendiaria de la Comuna de París, sino que dicha peligrosa fatalidad anímica abarcaría también a la misantropía de la literatura naturalista y a los libros de Schopenhauer (cf. p. 15). Precisamente en este crucial punto cabe suponer una cierta distancia crítica tomada por Nietzsche con respecto a la exposición de Bourget, a la que opone una comprensión distinta y más adecuada, a mi juicio, del papel desempeñado por la poética decadente de Baudelaire en la historia del pesimismo europeo del siglo XIX. Ya un provisorio examen de los diversos matices que adquiere el concepto de tedio y de su también problemática conexión con los ámbitos semióticos de lo que -desde un énfasis cuyo origen desciende hasta los inicios del romanticismo- se denomina lo monstruoso, podría clarificar el significado de esta divergencia que motiva la interpretación nietzcheana del pesimismo.

Bourget 1883, 13: "La force par laquelle nous persévérons dans l'existence est bornée et la puissance des causes extérieures la surpasse infiniment..." 
No parece difícil reconstruir un verdadero bestiarum monstrorum a partir del texto de Las flores del $\mathrm{mal}^{16}{ }^{16}$ pues en sus páginas los hay de diversa índole, sobresaliendo entre ellos los deformes (85), bicéfalos (115), viscosos (243), torcidos (disloqués) y fracturados (264), como así también los asesinos (323). En ocasiones, por ejemplo en el poema femmes damnées, la monstruosidad habita en los infiernos donde comparte protagonismo con vírgenes, demonios y mártires en el marco artístico del misticismo erótico y delirante que agobia al san Antonio de la obra pictórica del Bosco (313). Por otra parte, a diferencia de una prolongada opinión estética pre-romántica para la que lo monstruoso manifiesta exactamente lo opuesto a la armonía de lo bello, Baudelaire exclama -en un himno ofrendado a su nombre- que la belleza misma no es sino un monstruo enorme, pavoroso e ingenuo (117), ${ }^{17}$ con lo cual su poética sugiere ese locus suspectus o das Unheimliche que se afilia a la tradición de E.T.A. Hoffmann y Schelling, clasificada más tarde por la taxonomía psicoanalítica en la obra de Freud así titulada, alcanzando por ello una reputación conceptual que los intentos románticos-decadentes no pudieron haber siquiera atisbado. Pero sin duda que el más célebre de los habitantes de este universo paralelo es el tedio (l'Ennui), ese monstruo delicado enunciado ya -en complicidad lúdica con el lector- al comienzo de Las flores del mal y que Théophile Gautier describe en la introducción como le gran monstre moderne (cf. p.30).

Nietzsche se percata con razón pero demasiado tarde de que el apremio por investigar el sentido radical del tedio en la culminación de su siglo pasaba necesariamente por un examen riguroso de la obra de Kierkegaard, especialmente de los textos sobre la ironía, sobre la angustia y del ensayo titulado $O$ lo uno o lo otro, ${ }^{18}$ pero la lectura de Baudelaire sí lo mueve a indagar aún a tiempo los antecedentes de este monstruo moderno en una obra fragmentaria ya largamente conocida y muy apreciada, a saber, los Pensamientos de Pascal ${ }^{19}$. Allí se expone una comprensión del estado de aburrimiento que va muchísimo más allá de entenderlo al modo de una elemental disposición anímica, descubriendo en él más bien a una parte integrante de la Condition de l'homme, lo cual le permite a Pascal componer la siguiente descripción: "Tedio. - Nada tan insoportable para el hombre como estar en reposo total, sin pasiones, sin asuntos, sin diversiones, sin empleos. Él siente entonces su nada (Il sent alors son néant), su abandono, su insuficiencia, su dependencia, su impotencia, su vacío. Al instante extraerá del fondo de su alma el tedio, la negrura (noirceur), la tristeza, el pesar, el despecho, la desesperación" (Pascal 1962: 100).

Si se resta cierto patetismo que inevitablemente suena muy ajeno a los oídos contemporáneos, entonces se podrá coincidir, sin embargo, que en este pasaje se

16 A continuación cito la paginación de Les fleurs du mal de la edición de 1882 (cf. bibliografía) que Nietzsche poseía en su biblioteca (cf. Oehler 1942, 38) y que contiene un ensayo introductorio escrito por Théofile Gautier.

17 “O beauté! monstre énorme, effrayant, ingénu!".

18 El 19 de febrero de 1888, es decir, poco tiempo antes del colapso, comunica en una carta dirigida a Georg Brandes desde Niza que ha planificado dedicar su próxima estadía en Alemania al estudio de las obras tempranas de Kierkegaard, pues ha advertido que le serían de mucha utilidad, "en el mejor sentido de esta palabra”. Cf. KSB 8, 259.

19 El texto de Las flores del mal se ocupa de Pascal en el poema titulado El abismo, al que se hará referencia más adelante. En la edición que lee Nietzsche, además del ya nombrado ensayo de Gautier, se encuentra también entre los varios apéndices un comentario de Julius Barbey d'Aurevilly, autor estimado por él, al menos como ensayista (cf. KSB 8, 67), en el que se describe el pathos integral de Las flores del mal como comedia, pero como "una comedia de la que habla Pascal", es decir, "sangrienta" (cf. p. 370). 
encuentra ya parcialmente insinuada una cuestión que será central en el desarrollo de la filosofía cuando en las primeras décadas del siglo XX se intente indicar formalmente la estructura de la existencia desde el proceder fenomenológico y que bien podría añadirse a la fructíferas influencias que Kierkegaard y el propio Nietzsche han tenido evidentemente en ese proceso. Pero en el contexto de la reflexión decimonónica sobre el nihilismo europeo, los acentos no están aún puestos en un posible análisis existencial, por lo que el juicio pascaliano deberá dejarse someter todavía a la severidad de una forma de interrogación que siempre se origina en la sospecha frente a un aspecto del pesimismo cultural: aquel en que éste se descubre, en el fondo, como una negatividad al servicio de la escatología. Así lo expresa el discurso que sitia al hombre, a la naturaleza y a la historia de tal manera en el límite del nihil (o del abismo, en el caso de Pascal), que éste se ve luego casi forzado a aceptar la absoluta trascendencia de la promesa redentora que usualmente acompaña a ese mismo acoso radical que lo confina hasta las fronteras mismas de la aniquilación. Nietzsche cree poder verificar este procedimiento típico en un pasaje de los Pensamientos en que Pascal se interroga, afectado por el mismo pathos antes mencionado, sobre la fundamental incoherencia en que radica la Condition de l’homme: “QQué quimera es, pues, el hombre?" -se pregunta retóricamente, para responder en seguida mediante el recurso semántico de una figura paradojal- "iQué novedad, qué monstruo, qué caos, qué sujeto de contradicción, qué prodigio! juez de todas las cosas, imbécil gusano de tierra; depositario de lo verdadero, cloaca de incertidumbre y de error; gloria y desecho del universo" (212).

La pérdida de centralidad cosmológica y el nuevo concepto de infinitud derivados del giro copernicano son la impronta de modernidad que subyace a la apología pascaliana: lejos de querer demostrar la existencia de Dios mediante axiomas aristotélicos o a través del ontologismo neoplatónico, lo que ella propone es más bien una despiadada imagen de lo intolerable que se podría volver el universo y la vida del hombre si acaso se les privara del fundamento causal trascendente que explica tanto su origen como su finalidad. En un fragmento redactado en el otoño del año 1887, Nietzsche previene no sólo sobre el artificio ad horrendum inherente a la argumentación pascaliana, sino que muestra de qué manera este ultimátum apologético tuvo una continuidad ilustrada y se prolongó además secularizadamente hasta la filosofía de su siglo: “... 'sin la fe cristiana, opinaba Pascal, vosotros mismos, como también la naturaleza y la historia, os volveréis un monstre et un chaos'. Esta profecía nosotros la hemos cumplido: después de que el débil y optimista siglo XVIII hubiera embellecido y racionalizado al hombre. Schopenhauer y Pascal: en un sentido esencial, Schopenhauer es el primero que recoge el movimiento de Pascal: un monstre et un chaos, por consiguiente algo que hay que negar... la historia, la naturaleza, el hombre mismo. Nuestra incapacidad de conocer la verdad es la consecuencia de nuestra corrupción, de nuestra caída moral: así Pascal. Y así en el fondo Schopenhauer. 'cuando más profunda la corrupción, tanto más necesaria la doctrina de salvación' - o, dicho schopenhauerianamente, la negación.” (KSA 12, 445)

Aun cuando se podría profundizar más en este paso del pesimismo estético de la poética de Baudelaire a aquel que con Pascal y Schopenhauer podríamos calificar de pesimismo ontológico, baste en este contexto precisar que Nietzsche los vuelve a reunir en la caracterización de la negatividad que se opone al pesimismo clásico -al cual había dedicado su obra temprana- y que califica de pesimismo "romántico" (cf. 
KSA 13, 229), entendiéndolo, al mismo tiempo, como una huída estetizante del vacío tedioso que se mantiene, pues, sólo en el ámbito de la décadence, en la caída que parece hundirse siempre cada vez más sin topar empero nunca en fondo alguno, ya que en algún sentido se complace en este puro caer, en la debilidad de su condición que se lamenta en relación a la nada, pero que al mismo tiempo es incapaz de pensar radicalmente el nihil.

\section{OBRAS CITADAS}

Baudelaire, Charles. 1887. Oeuvres posthumes et correspondances inédites. Paris: Editorial Maison Quantin.

. 1882. Les Fleurs du mal. Précedées d'une notice par Théophile Gautier. Paris: Editorial Calman-Lévy Éditeurs.

Behler, Ernst. 1993. Studien zur Romantik und zur idealistischen Philosophie. Sokrates und die griechische Tragödie. Nietzsche und die bruder Schlegel über den Ursprung der Moderne. Paderborn: Editorial Schöningh.

Borget Paul. 1883. Essai de psychologie cotemporaine. Paris: Alphonse Lemerre, Éditor

Gawoll, Hans Jürgen. 1989. Nihilismus und Metaphysik. Entwicklungsgeschichte Untersuchung vom deitschen Idealismus bis zu Heidegger. Stuttgart: Editorial Frommann-Holzboog.

Le Rider, Lacques. 1997. Nietzsche in Frankreich. Paderborn: Editorial Wilhelm Fink.

Nietzsche, Friedrich. Kritische Studienausgabe (=KSA). 1967 y ss. Editada por Giorgio Colli y Mazzino Montinari. Berlín/New York: Editorial Walter de Gruyter (15 volúmenes)

Müller-Lauter, Wolfgang. 1999. Über Freiheit und Chaos. Nietzsche Interpretationen II. Berlín/New York: Editorial Walter de Gruyter. . 1971. Nietzsche. Seine Philosophie der Gegensätze und die Gegensätze seiner Philosophie. Berlín/New York: Editorial Walter de Gruyter.

Pascal. 1962. Pensées. Texte établi et annoté par Jacques Chevalier. Paris: Editorial Gallimard, Le livre de poche.

Pestalozzi, Karl. 1978. "Nietzsche Baudelaire-Rezeption“. Nietzsche-Studien 7, p. 158-188.

Volpi, Franco 2005. "Le nihilisme comme logique de la décadence. Nietzsche lecteur de Bourget”. Jean Francois Mattéi. Nietzsche et le temps des nihilismes. París: Editorial P.U.F. 